\title{
Absolute NMR shielding scales in methyl halides obtained from experimental and calculated nuclear spin-rotation constants
}

\author{
Daniel F. E. Bajac, I. Agustín Aucar, and Gustavo A. Aucar* \\ Instituto de Modelado e Innovación Tecnológica, CONICET, \\ and Departamento de Física - Facultad de Ciencias Exactas y Naturales, \\ UNNE, Avda. Libertad 5460, W3404AAS, Corrientes, Argentina.
}

(Dated: June 25, 2021)

\begin{abstract}
The non-relativistic "Ramsey-Flygare relationship" is the most used procedure to obtain semiexperimental NMR absolute shieldings by a correspondence between NMR shieldings $(\boldsymbol{\sigma})$ and nuclear spin-rotation constants $(\boldsymbol{M})$. One of its generalizations to the relativistic framework is known as the M-V model, which was proposed few year ago by some of the authors of the present work, and right now only applied to linear molecules. This model includes terms that does not have nonrelativistic counterparts and also include the paramagnetic contribution to the NMR shielding of nuclei in free atoms. All this ensures that its results fit quite well with those of four-component (4c) calculations. The first application of the M-V model to non-linear molecules, like methyl halides or $\mathrm{CH}_{3} X$ molecules $(X=\mathrm{F}, \mathrm{Cl}, \mathrm{Br}$ and $\mathrm{I})$, is given here. The analysis of each electronic mechanism of $\boldsymbol{\sigma}$ shows that most of their electron correlation effects are strongly related with the same effects in $\boldsymbol{M}$. By including experimental data of $M$ in the $\mathrm{M}-\mathrm{V}$ model most of the correlation effects are accurately taken into account for the absolute values of $\boldsymbol{\sigma}$. Calculations of $\boldsymbol{M}_{Y}$ and $\boldsymbol{\sigma}_{Y}(Y=$ $\mathrm{H}, \mathrm{C}$ and $X$ ) were carried out within the linear response formalism at the random phase level of approach and density functional theory in both, $4 \mathrm{c}$ and non-relativistic frameworks. The best fit between calculations of $\boldsymbol{M}$ and experimental data are obtained from calculations at 4c-PBE0 level of theory in all cases, but not for $M_{\|, C l}$ which suggest that a revision of the available experimental data may be necessary. There is an additional advantage of using $\mathrm{M}-\mathrm{V}$ model. One can indirectly calculate shieldings of open-shell free atoms, which cannot be obtained at the moment by applying 4c methods.
\end{abstract}

\section{INTRODUCTION}

Nuclear spin-rotation (SR) tensors $(\boldsymbol{M})$ are accurately measured by microwave spectroscopy. They were extensively employed to validate many-electron structure calculations in a wide number of molecules, and are quite useful in nuclear magnetic resonance (NMR) spectroscopy. The absolute values of NMR magnetic shieldings $(\boldsymbol{\sigma})$-which are very difficult to measure- are obtainable from them by applying the widely known "RamseyFlygare relationship" [1, 2]. This is a relationship between the paramagnetic contribution to the NMR shieldings and the electronic contribution to the SR constants within a non-relativistic (NR) framework.

During the last decade several groups of research around the world have made contributions that improve the calculation of the absolute shieldings in heavy-atom containing systems [3-13]. Four-component and twocomponent methodologies were applied, being one of the last ones, the M-V model developed by our group of research [14-16]. There are still some doubts about which methodology gives both, more insights on the physics behind and accurate results, given that one can use different strategies to generalize the Ramsey-Flygare relationship. On the other hand our model was only applied to linear systems and then it is necessary to enlarge its application to non-linear molecular systems in order to know whether

\footnotetext{
*gaaucar@conicet.gov.ar
}

such model can be safely used in non geometrically restricted molecular systems.

As mentioned, Flygare found an indirect way of obtaining absolute shielding constants by combining experimental SR data with some highly accurate calculations [17-19]. He assumed that:

1. The paramagnetic contributions to the shielding constants of free-atom nuclei are null.

2. The NMR shieldings and SR tensors for linear molecules have null elements on the symmetry axis of the molecule.

3. For molecules, there is an equivalence between the perturbative Hamiltonian that describes the interaction of magnetic moments of nuclear spins with an external and uniform magnetic field and the perturbative Hamiltonian that describes electronic effects due to molecular rotation of the nuclei.

Since the earliest theoretical works that included relativistic effects on NMR shieldings, it is known that the first statement is not correct. Besides, it is known that in a relativistic framework the symmetry axis elements of the NMR shielding tensor of a linear molecule are not zero, which violates condition $2[20]$. These findings together with some accurate experimental data collected by Wasylishen and collaborators suggested that the Ramsey-Flygare relationship is not valid when relativistic effects are not negligible [21]. More recently it was shown that the formal expressions of both properties -the NMR magnetic shielding and SR tensors- cannot 
be explicitly related to each other within the relativistic regime [22]. It means that it is not possible to obtain an exact theoretical relationship among them that would be valid within the relativistic framework. So, one of the above mentioned conditions, the third one, is not fulfilled any longer. Therefore, the Ramsey-Flygare relationship does not work in heavy-atom containing molecules.

One of the straight ways to generalize it makes use of the linear response within the elimination of small components (LRESC) model [23, 24]. The development of the M-V model was highly grounded on a bottom-up procedure whose first steps were worked out within the LRESC model. This procedure makes that, when the velocity of light, $\mathrm{c}$, is scaled to infinity, the M-V model goes to the Ramsey-Flygare one.[14, 15, 25]. It is also worth to mention that, in addition to the M-V model, some other models were recently developed in order to extend the applicability of the Ramsey-Flygare relationship to heavy-atom-containing molecules $[4,5]$.

All previous applications of the $\mathrm{M}-\mathrm{V}$ model to the computation of NMR shieldings have shown that it gives very close results to the four-component (4c) ones for heavyatom containing linear molecules $[15,16,26]$. As a next step of our development of model M-V we shall show here how accurate it is to reproduce magnetic shieldings in little more general molecules like the set $\mathrm{CH}_{3} X\left(X={ }^{19} \mathrm{~F}\right.$, $\left.{ }^{35} \mathrm{Cl},{ }^{79} \mathrm{Br},{ }^{127} \mathrm{I}\right)$, meaning non-linear compounds. For them we also studied the electronic origin of both, electron correlation and relativistic effects on paramagneticlike (or e-e) and diamagnetic-like (or p-p) contributions to both, $\boldsymbol{\sigma}$ and $\boldsymbol{M}$ tensors.

The analysis of the SR constants of both nuclei, $X$ and $\mathrm{H}$ in $\mathrm{CH}_{3} X$ molecules $(X=\mathrm{F}, \mathrm{Cl}, \mathrm{Br}$ and $\mathrm{I}$ ) has been performed in several computational and experimental works. The earliest experimental works applied different techniques: i) a molecular-beam electric resonance spectrometer to obtain radio-frequency spectra for $\mathrm{CH}_{3} \mathrm{~F}$ [27], ii) a molecular-beam maser spectrometer to obtain high-resolution measurements of hyperfine structure on $\mathrm{CH}_{3} \mathrm{Cl}$ [28] and iii) a molecular-beam absorption spectrometer to obtain high-resolution microwave spectra for $\mathrm{CH}_{3} \mathrm{Cl}$ [29], $\mathrm{CH}_{3} \mathrm{Br}$ [30] and $\mathrm{CH}_{3} \mathrm{I}$ [31]. Besides, rotational constants of $\mathrm{CH}_{3} \mathrm{Br}$ and $\mathrm{CH}_{3} \mathrm{I}$ were obtained with the highest accuracy by combining data taken from rotational spectra and pure quadrupole resonances [32]. The rotational spectra of $\mathrm{CH}_{3} \mathrm{I}$ was also observed once more time and analyzed in the submillimeter-wave region, being these data combined with the microwave and millimeter-wave measurements to determine with high accuracy its rotational constants [33]. Then, combining data obtained from Doppler-free double-resonance technique with that of previous Fourier-transform infrared spectroscopy, the molecular constants of $\mathrm{CH}_{3} \mathrm{I}$ were again obtained [34]. At the same time the Ramsey-Flygare relationship given in Eq. (4) of Section IIB of this work was applied to obtain semi-experimental values of NMR shieldings for $\mathrm{F}$ [27], $\mathrm{Cl}$ [29], $\mathrm{Br}$ [30] and I [31].
More recently, the microwave spectra of $\mathrm{CH}_{3} \mathrm{Cl}$ was obtained using the Prague millimeter-wave semiconductor spectrometer [35], and furthermore the Lamb-dip technique was exploited to obtain sub-Doppler resolution, enabling the determination of SR constants with an accuracy that rivals that obtained by molecular-beam electric resonance measurements on $\mathrm{CH}_{3} \mathrm{~F}$ [36].

Concerning the NMR spectroscopy, K. Jackowski and coworkers have developed a remarkable methodology to get experimentally based absolute shielding values. They obtained absolute shieldings of ${ }^{1} \mathrm{H}$ and ${ }^{13} \mathrm{C}$ in $\mathrm{CH}_{3} \mathrm{~F}$ [37], $\mathrm{CH}_{3} \mathrm{Br}$ [38] and $\mathrm{CH}_{3} \mathrm{I}$ [39]. A systematic study of this experimental set-up can be found in Ref. [40].

On the theoretical side, relativistic effects on magnetic shieldings of methyl halides were first studied with different methodologies which only included spinorbit (SO) interactions [41-46]. A more general though two-component perturbative approximation for including relativistic effects on magnetic properties, the LRESC model [23, 24], was recently applied to calculate $\sigma\left({ }^{127} \mathrm{I}\right)$, $\sigma\left({ }^{79} \mathrm{Br}\right), \sigma\left({ }^{13} \mathrm{C}\right)$ and $\sigma\left({ }^{1} \mathrm{H}\right)$ in $\mathrm{CH}_{3} \mathrm{Br}$ and $\mathrm{CH}_{3} \mathrm{I}[47]$. This method allows for the consideration of a number of relativistic mechanisms others than the SO one. Another and related recent work was devoted to the study of $\sigma\left({ }^{1} \mathrm{H}\right)$ and $\sigma\left({ }^{13} \mathrm{C}\right)$ for the family of $\mathrm{CH}_{3} X(X=\mathrm{F}, \mathrm{Cl}, \mathrm{Br}$ and I) molecules. Quantum chemical calculations were carried out at both, $a b$ initio and density functional theory (DFT) levels of approach, where relativistic corrections were taken into account at the leading-order Breit-Pauli perturbation level of approach [48].

To our knowledge there are only three papers that contain $4 \mathrm{c}$ calculations of $\sigma$ in methyl halides. In the first one, $\sigma\left({ }^{13} \mathrm{C}\right)$ in $\mathrm{CH}_{3} \mathrm{I}$ at $4 \mathrm{c}-\mathrm{DFT}$ (using the KT3 functional) and random phase approximation (RPA) levels of theory, and using the gauge-independent atomic orbital (GIAO) scheme were studied. The authors also calculated $\sigma\left({ }^{13} \mathrm{C}\right)$ with a hybrid method, mixing NR-MP2 and 4c-RPA calculations [49]. Then, in a second paper the same authors extended their work to the analysis of $\sigma\left({ }^{13} \mathrm{C}\right)$ in $70 \mathrm{CX}_{n} Y_{4-n}$ halogenomethanes (with $n=1$, 2, 3, 4 and $X, Y=\mathrm{H}, \mathrm{F}, \mathrm{Cl}, \mathrm{Br}$ and I). They performed RPA and DFT 4c calculations (with the OPW91 functional), and also used two hybrid methods (NR-MP2 + 4c-RPA and NR-MP2 + 4c-OPW91) to calculate $\sigma\left({ }^{13} \mathrm{C}\right)$ [50]. The third one is the most recent and is close to the present one [13]. Uhlíková and coauthors have calculated $\sigma(\mathrm{Br})$ and $\sigma(\mathrm{I})$ in $\mathrm{CH}_{3} \mathrm{Br}$ and $\mathrm{CH}_{3} \mathrm{I}$ using a $4 \mathrm{c}$ DiracCoulomb Hamiltonian at DFT-BP86 and DFT-PBE levels of approach, together with their NR counterparts. They compared the results of calculations of shieldings and SR constants employing experimental and $a b i n i$ tio optimized equilibrium geometries, but then they applied the Ramsey-Flygare relationship to get $\sigma$, employing both, NR and 4c methods. Another aim of our work is related with the analysis of the consequences that arise on the values of absolute shieldings when they are calculated in this way.

In the following section we treat the equations for NMR 
shielding and nuclear SR tensors derived within the $4 \mathrm{c}$ framework, as well as the relationship between them, both in NR and 4c levels of theory. In Section III the computational details for all calculations are given and Section IV start with with a comparison of calculated nuclear SR tensor elements with the corresponding experimental data. The accuracy of the M-V model for methyl halides is then analyzed and concluding remarks are given in Section V.

\section{THEORY}

The SR tensor of a nucleus $Y, \boldsymbol{M}_{Y}$, couples the magnetic dipole moment due to nuclear spin with the molecular rotational angular momentum [19, 22]. On the other hand, the NMR shielding tensor of a nucleus $Y, \boldsymbol{\sigma}_{Y}$, couples the nuclear magnetic dipole moment with an external uniform magnetic field.

\section{A. Nuclear spin-rotation and NMR shielding tensors}

The tensor $\boldsymbol{M}_{Y}$ in a molecule in equilibrium depends on both, nuclear and electronic degrees of freedom: $\boldsymbol{M}_{Y}$ $=\boldsymbol{M}_{Y}^{\text {nuc }}+\boldsymbol{M}_{Y}^{\text {elec }}[19,22]$. In Gaussian atomic units, which are used throughout the present work, they are written as

$\boldsymbol{M}_{Y}^{n u c}=\sum_{X \neq Y} \frac{g_{Y} Z_{X}}{2 m_{p} c^{2}}\left[\boldsymbol{R}_{X, C M} \cdot \frac{\boldsymbol{R}_{X Y}}{\left|\boldsymbol{R}_{X Y}\right|^{3}}-\boldsymbol{R}_{X, C M} \frac{\boldsymbol{R}_{X Y}}{\left|\boldsymbol{R}_{X Y}\right|^{3}}\right] \otimes$

and

$$
\left.\boldsymbol{M}_{Y}^{\text {elec }}=\frac{g_{Y}}{2 m_{p} c^{2}}\left\langle\left\langle\left(\frac{\boldsymbol{r}-\boldsymbol{R}_{Y}}{\left|\boldsymbol{r}-\boldsymbol{R}_{Y}\right|^{3}} \times c \boldsymbol{\alpha}\right) ; \boldsymbol{J}_{e}\right\rangle\right\rangle \otimes \boldsymbol{I}^{-} \nmid 2\right)
$$

Here, $g_{Y}$ is the nuclear g-value of nucleus $Y, Z_{X}$ is the atomic number of nucleus $X, m_{p}$ is the proton mass, $c$ is the speed of light in vacuum, $\boldsymbol{R}_{X Y}$ and $\boldsymbol{R}_{X, C M}$ are the position vectors of nucleus $X$ with respect to nucleus $Y$ and the molecular center of mass, respectively, and $\boldsymbol{I}$ is the molecular moment of inertia tensor in the equilibrium geometry with respect to its center of mass. Furthermore, $\langle\langle;\rangle\rangle$ stands for the relativistic polarization propagator, $\boldsymbol{\alpha}$ is the Dirac operator, and $\boldsymbol{J}_{e}=\left(\boldsymbol{r}-\boldsymbol{R}_{C M}\right) \times \boldsymbol{p}+\frac{1}{2} \boldsymbol{\Sigma}$ is the relativistic electronic total angular momentum operator, which is the sum of the total orbital and spin angular momenta. The orbital angular momentum is taken with respect to the molecular center of mass, and $\boldsymbol{\Sigma}$ is the $4 \mathrm{c}$ extension of the Pauli matrices. Then, Eq. (2) can be written as $\boldsymbol{M}_{Y}^{\text {elec }}=\boldsymbol{M}_{Y}^{L}+\boldsymbol{M}_{Y}^{S}$.

On the other hand, within the 4c polarization propagator theory (4c-PolProp) $\boldsymbol{\sigma}_{Y}$ is written as

$$
\boldsymbol{\sigma}_{Y}=\frac{1}{2 c^{2}}\left\langle\left\langle\left(\frac{\boldsymbol{r}-\boldsymbol{R}_{Y}}{\left|\boldsymbol{r}-\boldsymbol{R}_{Y}\right|^{3}} \times c \boldsymbol{\alpha}\right) ;\left(\boldsymbol{r}-\boldsymbol{R}_{G}\right) \times c \boldsymbol{\alpha}\right\rangle\right\rangle,(3)
$$

where $\boldsymbol{R}_{G}$ represents the gauge origin position of the magnetic potential.
Working with the 4c-PolProp, tensors $\boldsymbol{M}_{Y}^{\text {elec }}$ and $\boldsymbol{\sigma}_{Y}$ can be approximated as the sum of two terms which contain the following transition moment matrix elements: one that is built between occupied positive-energy orbitals and unoccupied positive-energy orbitals (e-e contributions), and another one that is built between occupied positive-energy orbitals and unoccupied negative-energy orbitals (p-p contributions) [51, 52].

\section{B. Relationship between $\sigma$ and $M$}

Ramsey and Flygare based their works on the Schrödinger representation. They found a reliable theoretical relationship between $\boldsymbol{\sigma}_{Y}$ and $\boldsymbol{M}_{Y}$ which is still useful for obtaining absolute shieldings from measured SR constants $[1,2,19]$. Such a relation, the RamseyFlygare relationship, is valid when the molecules do not contain heavy atoms and are considered as rigid rotors; it is written as

$$
\begin{aligned}
\boldsymbol{\sigma}_{Y} & =\boldsymbol{\sigma}_{Y}^{N R-p a r a}+\boldsymbol{\sigma}_{Y}^{N R-d i a} \\
& \approx \frac{m_{p}}{g_{Y}} \boldsymbol{M}_{Y}^{N R} \otimes \boldsymbol{I}+\boldsymbol{\sigma}_{Y}^{F A, N R} .
\end{aligned}
$$

The symbols $\boldsymbol{\sigma}_{Y}^{N R-p a r a}$ and $\boldsymbol{\sigma}_{Y}^{N R-d i a}$ stand for paramagnetic and diamagnetic contributions to the NR shielding, respectively, and $\boldsymbol{\sigma}_{Y}^{F A, N R}$ is the shielding of a nucleus $Y$ for a free atom (which has only diamagnetic contributions within the NR domain). Furthermore, results of calcu1 lations with the Ramsey-Flygare relationship are more accurate for isotropic values than for individual tensor elements [17-19].

Our main concern here is related with the application of the M-V model to non-linear molecules. This model is the most accurate of the set of M-i ( $\mathrm{i}=\mathrm{I}$ to $\mathrm{V}$ ) models whose theoretical grounds are given elsewhere $[15,16]$. In this model both properties are related as follows:

$$
\boldsymbol{\sigma}_{Y}^{M-V}=\boldsymbol{\sigma}_{Y}^{S R}+\boldsymbol{\sigma}_{Y}^{F A}+\frac{1}{2 c}\left(\boldsymbol{\nu}_{Y}^{S}-\boldsymbol{\nu}_{Y}^{F A, S}\right) .
$$

where

$$
\boldsymbol{\sigma}_{Y}^{S R}=\frac{m_{p}}{g_{Y}} \boldsymbol{M}_{Y} \otimes \boldsymbol{I},
$$

$\boldsymbol{\sigma}_{Y}^{F A}$ is the 4c shielding tensor of nucleus $Y$ for the free atom, and both $\boldsymbol{\nu}_{Y}^{S}$ and $\boldsymbol{\nu}_{Y}^{F A, S}$ are terms without NR equivalents, where[15]

$$
\frac{1}{2 c} \boldsymbol{\nu}_{Y}^{S}=\frac{1}{2 c^{2}}\left\langle\left\langle\left(\frac{\boldsymbol{r}-\boldsymbol{r}_{Y}}{\left|\boldsymbol{r}-\boldsymbol{r}_{Y}\right|^{3}} \times c \boldsymbol{\alpha}\right) ; \boldsymbol{S}_{e}\right\rangle\right\rangle=\frac{m_{p}}{g_{Y}} \boldsymbol{M}_{Y}^{S} \otimes \boldsymbol{I},
$$

being $\boldsymbol{S}_{e}=\frac{1}{2} \boldsymbol{\Sigma}$. Besides, $\boldsymbol{\nu}_{Y}^{F A, S}$ has the same expression as that of $\boldsymbol{\nu}_{Y}^{S}$, but refers to the free atom. In addition, the linear response on the rhs of Eq. (7) is formally the same as that of $M_{Y}^{S}$.

One can easily see that Eqs. (4) and (5) are expressed with some similar formal terms. The main difference is 
that the first one is written within the NR framework, meaning that it includes only scalar terms; instead, the model $\mathrm{M}-\mathrm{V}$ have all its elements written within the relativistic framework.
We shall consider two different components of the $\boldsymbol{\sigma}$ tensors for carbon and halogens nuclei. They are the perpendicular and parallel components relative to the $\mathrm{C}$ $X$ molecular bond axis. Hence,

$$
\sigma_{\perp(\|), Y}^{M-V(e-e)}=\frac{m_{p} I_{\perp(\|)}}{g_{Y}} M_{\perp(\|), Y}^{e l e c(e-e)}+\sigma_{Y}^{F A(e-e)}+\frac{1}{2 c}\left(\nu_{\perp(\|), Y}^{S(e-e)}-\nu_{Y}^{F A, S(e-e)}\right)
$$

and

$$
\sigma_{\perp(\|), Y}^{M-V(p-p)}=\frac{m_{p} I_{\perp(\|)}}{g_{Y}}\left(M_{\perp(\|), Y}^{n u c}+M_{\perp(\|), Y}^{e l e c(p-p)}\right)+\sigma_{Y}^{F A(p-p)}++\frac{1}{2 c}\left(\nu_{\perp(\|), Y}^{S(p-p)}-\nu_{Y}^{F A, S(p-p)}\right)
$$

Then, the isotropic shielding constant for $\mathrm{H}, \mathrm{C}$ and $X$ nuclei are expressed as

$$
\sigma_{i s o, Y}^{M-V}=\sigma_{i s o, Y}^{S R}+\sigma_{Y}^{F A}+\frac{1}{2 c}\left(\nu_{i s o, Y}^{S}-\nu_{Y}^{F A, S}\right)
$$

\section{COMPUTATIONAL DETAILS}

Gas phase experimental geometries, determined by microwave and infrared spectroscopies and taken from Ref. [53], were considered for $\mathrm{CH}_{3} X\left(X={ }^{19} \mathrm{~F},{ }^{35} \mathrm{Cl}\right.$, $\left.{ }^{79} \mathrm{Br},{ }^{127} \mathrm{I}\right)$ molecules. The equilibrium bond distances and angles are given in Table I.

TABLE I: Equilibrium bond distances and angles for $\mathrm{CH}_{3} X\left(X={ }^{19} \mathrm{~F},{ }^{35} \mathrm{Cl},{ }^{79} \mathrm{Br},{ }^{127} \mathrm{I}\right)$ molecules.

\begin{tabular}{lcccc}
\hline & $\mathrm{CH}_{3} \mathrm{~F}$ & $\mathrm{CH}_{3} \mathrm{Cl}$ & $\mathrm{CH}_{3} \mathrm{Br}$ & $\mathrm{CH}_{3} \mathrm{I}$ \\
\hline $\mathrm{C}-\mathrm{H}($ in $\AA)$ & 1.095 & 1.090 & 1.086 & 1.084 \\
$\mathrm{C}-X($ in $\AA)$ & 1.382 & 1.785 & 1.933 & 2.132 \\
$\theta(\mathrm{HCH})$ & $110.45^{\circ}$ & $110.8^{\circ}$ & $111.2^{\circ}$ & $111.2^{\circ}$ \\
\hline
\end{tabular}

Furthermore, calculations of SR constants were performed with values of nuclear g-factors taken from Ref. [54]: 5.585694 for ${ }^{1} \mathrm{H}, 1.404824$ for ${ }^{13} \mathrm{C}, 5.257736$ for ${ }^{19} \mathrm{~F}, 0.547916$ for ${ }^{35} \mathrm{Cl}, 1.404267$ for ${ }^{79} \mathrm{Br}$ and 1.125309 for ${ }^{127}$ I. Four-component calculations of $\boldsymbol{\sigma}, \boldsymbol{M}$ and $\boldsymbol{\nu}^{S}$ were performed with the DIRAC program package [55, 56]. All of them were based on the Dirac-Coulomb Hamiltonian, employing the default choice for a Hamiltonian of the DIRAC code. It uses an energy correction to avoid the explicit calculation of (SS|SS) integrals [57].

In all calculations, Dyall's relativistic acv4z basis sets (dyall.acv4z) were employed for hydrogen (K. G. Dyall, unpublished), fluorine, carbon, chlorine [58], bromine and iodine [59], together with uncontracted Gaussian basis sets and the common gauge-origin (CGO) approach (additional calculations employing GIAOs are given in Supplemental Material [60]). The small component basis sets for relativistic calculations were generated by applying the unrestricted kinetic balance prescription (UKB)
[52]. In addition, the Gaussian nuclear charge distribution was used [61].

Most of response calculations have been carried out at the 4c-PolProp/RPA level of approach employing Dirac Hartree-Fock (DHF) wave functions. Non-relativistic values of $\boldsymbol{\sigma}$ and $\boldsymbol{M}$ (reported here as $\boldsymbol{\sigma}^{N R-\text { para }}, \boldsymbol{\sigma}^{N R-\text { dia }}$ and $\boldsymbol{M}^{N R-\text { elec }}$ ) were obtained scaling the speed of light to $c=100 c_{0}$. The value of the speed of light in vacuum used throughout all $4 \mathrm{c}$ calculations was $c_{0}=137.0359998$ a.u..

The gauge origin for the external magnetic potential was always placed at the molecular center of mass. Then a direct comparison with the SR results can be safely made. Furthermore, the values of $\sigma^{F A}$ and $\nu^{F A, S}$ were calculated for the following anions: ${ }^{19} \mathrm{~F}^{-},{ }^{35} \mathrm{Cl}^{-},{ }^{79} \mathrm{Br}^{-}$ and ${ }^{127} \mathrm{I}^{-}$, instead of the neutral atoms in order to consider closed-shell systems.

The influence of electron correlation effects was studied through Dirac Kohn-Sham (DKS) DFT calculations performed employing the DIRAC code. DFT calculations are also based on the 4c Dirac-Coulomb Hamiltonian and have been done using a variety of NR exchangecorrelation functionals in the following categories: (i) the generalized gradient approximation (GGAs), functionals KT3 [62] and BP86 [63, 64], and (ii) the hybrid functional PBE0 [65].

\section{RESULTS AND DISCUSSION}

In this Section, 4c calculations of SR constants at RPA and DFT levels of theory are compared with available experimental data for hydrogen and halogen atoms. Afterwards, each term of $\boldsymbol{\sigma}_{Y}^{M-V}$ is analyzed separately in order to learn about the underlying physics that may be influencing both, the paramagnetic-like (e-e) and diamagnetic-like (p-p) contributions to $\boldsymbol{\sigma}_{Y}$. In line with this, results of $4 \mathrm{c}$ calculations of the isotropic shieldings are compared with those obtained from the application of the M-V model. Then, an analysis of semi-experimental values of absolute shieldings is introduced, where experimental SR constants are included into the $\mathrm{M}-\mathrm{V}$ 
model together with highly accurate calculations of $\sigma_{Y}^{F A}$ and $\frac{1}{2 c}\left(\nu_{i s o, Y}^{S}-\nu_{Y}^{F A, S}\right)$ to obtain values of $\sigma_{i s o, Y}^{M-V}$ (semiexpt). In the last subsection we shall give an analysis of the electronic origin of $\boldsymbol{M}_{C}$ and $\boldsymbol{\sigma}_{C}$ for ${ }^{13} \mathrm{C}$.

\section{A. Four component calculations of the tensor $M$}

Results of calculations of parallel and perpendicular components of $\boldsymbol{M}_{X}$, and the isotropic contribution to $\boldsymbol{M}_{H}$ are displayed in Table II. We start analyzing $\boldsymbol{M}_{X}$. As expected, relativistic effects in $\boldsymbol{M}_{X}$ increase as $\mathrm{Z}_{X}$ becomes higher. Besides, those effects have opposite signs on the perpendicular and parallel tensor elements. This fact makes that relativistic effects on isotropic values of $\boldsymbol{M}_{X}$ become smaller than the ones in each component.

Results of calculations at 4c-PBE0 level of theory are, by far, the best fitting to experimental data. Those values are within error bars of experiments for both, perpendicular and parallel components of the nuclear spinrotation tensor and for all nuclei, but not for the chlorine nucleus in $\mathrm{CH}_{3} \mathrm{Cl}$. For this last case a discrepancy between calculated and experimental values is found, even if the experimental error bar is particularly large. So it may be interesting to know whether new measurements can confirm which one of both numbers is the most accurate.

Furthermore, only 4c calculations reproduce in an adequate manner the experimental anisotropies of the SR tensor of halogens, i.e. the differences between $M_{\perp, X}$ and $M_{\|, X}$, as it can be seen from results given in Table II. In order to be more clear about this fact, we take the iodine nucleus as an example. The experimental value of its anisotropy is $(-0.298 \pm 0.097) \mathrm{kHz}$, whereas the NRPBE0 and 4c-PBE0 calculations give $-3.4299 \mathrm{kHz}$ and $-0.2693 \mathrm{kHz}$, respectively.

Concerning electron correlation our results show that they are larger than the relativistic effects in all cases. These last ones are less than $1.6 \mathrm{kHz}$ for $M_{\perp, X}$ and up to $3.0 \mathrm{kHz}$ for $M_{\|, X}$. On the other hand, correlation effects are $6.0 \mathrm{kHz}$ at most for both perpendicular and parallel components of $\boldsymbol{M}_{X}$.

Turning now to the analysis of the isotropic values of $\boldsymbol{M}_{H}$ we want first to highlight that both effects, relativistic and electron correlation, are very small. This behavior is different to the one observed in hydrogen halides. In these last molecules the $H A L A$ effects (due to the inclusion of relativity in the calculations) strongly contribute to $\boldsymbol{M}_{H}$ [66]. This means that, for methyl halides, heavyatom effects are not efficiently transmitted through twobonds. Again electron correlation effects are higher than the relativistic ones. On the other hand, electron correlation effects grows up in the opposite direction as compared to the relativistic effects, meaning from $\mathrm{CH}_{3} \mathrm{I}$ to $\mathrm{CH}_{3} \mathrm{~F}$.

\section{B. Accuracy of the M-V model}

Since 2016, the M-V model was successfully applied to a few set of linear molecules which include the following dihalogen molecules: $X Y(X, Y=\mathrm{H}, \mathrm{F}, \mathrm{Cl}, \mathrm{Br}, \mathrm{I}, \mathrm{At})$ [15], and some others like $U V(U=\mathrm{Li}, \mathrm{Na}, \mathrm{K}, \mathrm{Rb}, \mathrm{Cs}$, $\mathrm{Cu}, \mathrm{Ag}, \mathrm{Au}, \mathrm{H}, \mathrm{F}, \mathrm{Cl}, \mathrm{Br}, \mathrm{I} ; V=\mathrm{H}, \mathrm{I}), \operatorname{Ag} Z$ and $\mathrm{Cs} Z(Z$ $=\mathrm{H}, \mathrm{F}, \mathrm{Cl}, \mathrm{Br}, \mathrm{I})[16]$. In those cases, NMR shielding constants were accurately reproduced. So, if the same happens also for methyl halides it would mean that our model could also be safely applied to some non-linear molecules.

In Table III perpendicular and parallel tensor elements of $\boldsymbol{\sigma}_{X}$, in terms of (e-e) and (p-p) contributions are given. The same occurs with $\boldsymbol{\sigma}_{X}^{M-V}$. The differences among the (e-e) contributions to $\boldsymbol{\sigma}_{X}$ and $\boldsymbol{\sigma}_{X}^{M-V}$ increase as $Z_{X}$ grows, whereas the opposite trend is found for the differences between their (p-p) contributions. They are less than $1.6 \mathrm{ppm}$ (in absolute values) for all halogen atoms. Besides, the M-V model better reproduces the values of $\sigma_{\|, X}^{(e-e)}$ than those of $\sigma_{\perp, X}^{(e-e)}$. Furthermore, in agreement with earlier works $[17,18]$, the differences in the (p-p) contributions of both components, i.e. $\sigma_{\perp, X}^{(p-p)}-\sigma_{\perp, X}^{M-V(p-p)}$ and $\sigma_{\|, X}^{(p-p)}-\sigma_{\|, X}^{M-V(p-p)}$, have opposite signs. Then, the model M-V reproduces $\sigma_{i s o, X}^{(p-p)}$ better than their individual tensor elements. The same behavior is found in the NR Ramsey-Flygare relationship $[14,17-19]$. The behavior of the (e-e) contributions to $\boldsymbol{\sigma}_{X}$ and to each one of the three terms of the rhs of Eq. (5) are shown in Fig. 1. According to the Ramsey-Flygare relationship, the NR limit of $\boldsymbol{\sigma}_{X}^{(e-e)}$ (which is equal to $\boldsymbol{\sigma}_{X}^{N R-p a r a}$ ) is such that the last two terms of that equation are zero. This fact allows for to realize that relativistic effects in $\boldsymbol{M}_{X}^{(e-e)}$ are much smaller than those in $\boldsymbol{\sigma}_{X}^{(e-e)}$ (see Fig. 1). The highest relativistic effects (in percentage) appear for $M_{\|, I}^{(e-e)}(12.8 \%)$ followed by $M_{\perp, I}^{(e-e)}(10.4 \%)$. Besides, for all cases other than iodine the relativistic effects in $M_{\perp, X}^{(e-e)}$ and $M_{\|, X}^{(e-e)}$ are smaller than $5 \%$.

In addition, it is clearly seen that relativistic effects in $\boldsymbol{\sigma}_{X}^{(e-e)}$ grow up from fluorine to iodine, and this occurs for both, perpendicular and parallel tensor components. In particular, relativistic effects in $\sigma_{\|, I}^{(e-e)}$ contribute around $1662.00 \mathrm{ppm}$. According to the M-V model, this quite large value (its NR limit is $\sigma_{\|, I}^{N R-\text { para }}=-62.55 \mathrm{ppm}$ ) is mainly due to the core contribution of $\sigma_{I^{-}}^{F A(e-e)}$, whose NR limit is equal to zero.

In order to highlight the accuracy of our model we performed a deeper analysis of the shielding of iodine nucleus in methyl iodide. Calculations of $\frac{m_{p} I}{g_{I}}\left(M_{\perp, I}^{(e-e)}-M_{\perp, I}^{N R-e l e c}\right), \quad \sigma_{I^{-}}^{F A(e-e)}$ and $\frac{1}{2 c}\left(\nu_{\perp, I}^{S(e-e)}-\nu_{I^{-}}^{F A, S(e-e)}\right)$ at RPA level of approach give 
TABLE II: Results (in $\mathrm{kHz})$ of $\mathrm{NR}$ and $4 \mathrm{c}$ calculations of $M_{\perp}(X), M_{\|}(X)$ and $M_{\text {iso }}(\mathrm{H})$ for $\mathrm{CH}_{3} X$ molecules $(X=$ $\mathrm{F}, \mathrm{Cl}, \mathrm{Br}$ and I).

\begin{tabular}{|c|c|c|c|c|c|c|c|c|c|c|c|}
\hline \multirow[b]{2}{*}{ Molec } & \multirow[b]{2}{*}{ Nuc } & \multirow[b]{2}{*}{ Comp } & \multicolumn{4}{|c|}{ NR } & \multicolumn{4}{|c|}{$4 c$} & \multirow[t]{2}{*}{ Expt } \\
\hline & & & RPA & BP86 & PBE0 & KT3 & $\mathrm{RPA}$ & BP86 & PBE0 & KT3 & \\
\hline \multirow[t]{3}{*}{$\mathrm{CH}_{3} \mathrm{~F}$} & $\mathrm{~F}$ & $\perp$ & 6.2594 & 2.4380 & 3.7170 & 0.0005 & 6.2557 & 2.4360 & 3.7149 & -0.0134 & $4.50 \pm 0.58^{\mathrm{a}}$ \\
\hline & $\mathrm{F}$ & $\|$ & -45.0782 & -65.0642 & -57.4576 & -63.7227 & -45.2198 & -65.2510 & -57.6251 & -63.9125 & $-55.7 \pm 1.2^{\mathrm{a}}$ \\
\hline & $\mathrm{H}$ & iso & 5.6462 & 5.2498 & 5.3795 & 5.3746 & 5.6603 & 5.2676 & 5.3957 & 5.3933 & \\
\hline \multirow[t]{3}{*}{$\mathrm{CH}_{3} \mathrm{Cl}$} & $\mathrm{Cl}$ & $\perp$ & -1.8819 & -2.6344 & -2.3548 & -2.5833 & -1.8772 & -2.6332 & -2.3525 & -2.5854 & $-2.150 \pm 0.063^{b}$ \\
\hline & $\mathrm{Cl}$ & $\|$ & -3.6698 & -5.0344 & -4.5309 & -4.9215 & -3.7069 & -5.0948 & -4.5833 & -4.9827 & $-7.0 \pm 1.6^{\mathrm{c}}$ \\
\hline & $\mathrm{H}$ & iso & 5.6034 & 5.3640 & 5.4383 & 5.4873 & 5.6209 & 5.3881 & 5.4601 & 5.4874 & \\
\hline \multirow[t]{3}{*}{$\mathrm{CH}_{3} \mathrm{Br}$} & $\mathrm{Br}$ & $\perp$ & -10.1826 & -14.5261 & -12.9952 & -14.2724 & -9.8760 & -14.4345 & -12.8472 & -14.2405 & $-12.63 \pm 0.10^{\mathrm{d}}$ \\
\hline & $\mathrm{Br}$ & $\|$ & -14.3106 & -19.9670 & -17.9759 & -19.9978 & -15.0453 & -21.2517 & -19.0814 & -21.2982 & $-18.8 \pm 1.7^{\mathrm{d}}$ \\
\hline & $\mathrm{H}$ & iso & 5.5808 & 5.3708 & 5.4342 & 5.4653 & 5.6241 & 5.4388 & 5.4952 & 5.5365 & \\
\hline \multirow[t]{3}{*}{$\mathrm{CH}_{3} \mathrm{I}$} & $\mathrm{I}$ & $\perp$ & -14.8484 & -19.9219 & -18.0681 & -19.5614 & -13.2497 & -19.5386 & -17.4197 & -19.2467 & $-17.398 \pm 0.0475^{\circ}$ \\
\hline & I & $\|$ & -11.5564 & -16.1591 & -14.6382 & -16.4485 & -13.0986 & -19.0835 & -17.1504 & -19.4188 & $-17.10 \pm 0.085^{\mathrm{e}}$ \\
\hline & $\mathrm{H}$ & iso & 5.6514 & 5.5083 & 5.5236 & 5.5599 & 5.6915 & 5.5702 & 5.6111 & 5.6652 & \\
\hline
\end{tabular}

a Taken from Ref. [36].

b Taken from Ref. [35].

c Taken from Ref. [29].

d Taken from Ref. [30].

e Taken from Ref. [34]. Standard deviations computed as one half of the reported errors.

TABLE III: Calculated values (in ppm) of $\perp$ and $\|$ components of $\boldsymbol{\sigma}_{X}$ and $\boldsymbol{\sigma}_{X}^{M-V}$ in terms of their (e-e) and (p-p) contributions. Four-component calculations were performed at the RPA level of approach.

\begin{tabular}{|c|c|c|c|c|c|c|}
\hline & $\sigma_{X}^{(e-e)}$ & $\sigma_{X}^{M-V(e-e)}$ & $\Delta^{(e-e)}$ & $\sigma_{X}^{(p-p)}$ & $\sigma_{X}^{M-V(p-p)}$ & $\Delta^{(p-p)}$ \\
\hline \multicolumn{7}{|c|}{$X=\mathrm{F}$} \\
\hline$\perp$ & 6.14 & 6.40 & -0.26 & 505.19 & 519.11 & -13.92 \\
\hline$\|$ & -48.31 & -48.18 & -0.13 & 489.33 & 479.41 & 9.92 \\
\hline \multicolumn{7}{|c|}{$X=\mathrm{Cl}$} \\
\hline$\perp$ & -222.18 & -221.79 & -0.39 & 1157.30 & 1166.89 & -9.59 \\
\hline$\|$ & 9.66 & 9.76 & -0.10 & 1129.47 & 1125.35 & 4.12 \\
\hline \multicolumn{7}{|c|}{$X=\mathrm{Br}$} \\
\hline$\perp$ & -173.30 & -172.34 & -0.96 & 2956.06 & 2963.95 & -7.89 \\
\hline$\|$ & 411.54 & 411.78 & -0.24 & 2920.03 & 2916.75 & 3.28 \\
\hline \multicolumn{7}{|c|}{$X=\mathrm{I}$} \\
\hline$\perp$ & 516.04 & 517.64 & -1.60 & 4932.43 & 4939.19 & -6.76 \\
\hline$\|$ & 1599.67 & 1600.09 & -0.42 & 4894.33 & 4892.44 & 1.89 \\
\hline
\end{tabular}

(as shown in Fig. 1a) $171.73 \mathrm{ppm}, 1913.47 \mathrm{ppm}$ and $81.81 \mathrm{ppm}$, respectively. The addition of all of them gives $2167.01 \mathrm{ppm}$, and the relativistic effect in $\sigma_{\perp, I}^{(e-e)}$ (i.e. $\sigma_{\perp, I}^{(e-e)}-\sigma_{\perp, I}^{N R-p a r a}$ ) is $2165.41 \mathrm{ppm}$. Therefore, the difference between relativistic effects of $\sigma_{\perp, I}^{(e-e)}$ and $\sigma_{\perp, I}^{M-V(e-e)}$ amount to only $-1.60 \mathrm{ppm}$, meaning that it is less than $0.1 \%$ (as seen in Table III). Then, it can safely be stated that the model $\mathrm{M}-\mathrm{V}$ accurately reproduces the relativistic effects for $\sigma_{\perp, I}^{(e-e)}$.

Furthermore, the application of our model to the study of $\sigma_{\|, I}^{(e-e)}$ gives even better results. In Eq. (8) it is seen that relativistic effects arises from the following three factors: $\frac{m_{p} I}{g_{I}}\left(M_{\|, I}^{(e-e)}-M_{\|, I}^{N R-e l e c}\right), \sigma_{I^{-}}^{F A(e-e)}$ and $\frac{1}{2 c}\left(\nu_{\|, I}^{S(e-e)}-\nu_{I^{-}}^{F A, S(e-e)}\right)$. Their 4c-RPA values are $-8.25 \mathrm{ppm}, 1913.47 \mathrm{ppm}$ and $-242.80 \mathrm{ppm}$, respectively (see Fig. 1b), being the addition of the three equal to $1662.42 \mathrm{ppm}$, while $\sigma_{\|, I}^{(e-e)}-\sigma_{\|, I}^{N R-\text { para }}=1662.00 \mathrm{ppm}$. So, in this case the difference between them is $-0.42 \mathrm{ppm}$ (as reported in Table III).

Concerning the (p-p) contributions (see Eq. (9)), we observe that relativistic contributions to $\sigma_{\perp(\|), Y}^{(p-p)}$ are mainly given by those of $\sigma_{Y}^{F A(p-p)}$. In the special case of iodine we found that there is an accurate matching between $4 \mathrm{c}$ and the M-V values at the RPA level of approach (see Table III). Besides, $\sigma_{I^{-}}^{F A(p-p)}=4890.21 \mathrm{ppm}$, $\sigma_{\perp, I}^{(p-p)}=4932.43 \mathrm{ppm}$ and $\sigma_{\|, I}^{(p-p)}=4894.33 \mathrm{ppm}$, so that almost the whole contribution to $\sigma_{\perp(\|), I}^{(p-p)}$ is of an atomic nature. Therefore, we note that ( $p-p)$ contributions to shieldings are such that: (i) they are almost completely described by $\sigma^{F A(p-p)}$, and (ii) parallel and perpendicular tensor elements have values of $\boldsymbol{\sigma}^{(p-p)}-\boldsymbol{\sigma}^{M-V(p-p)}$ 


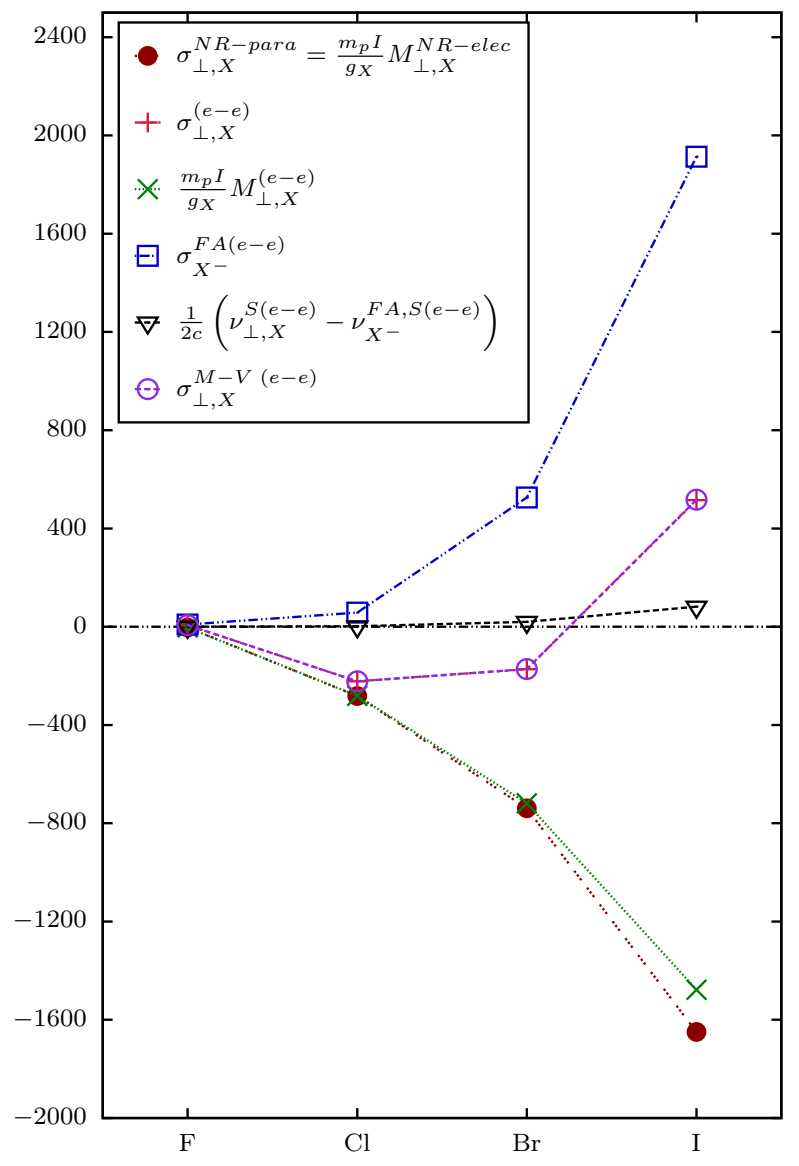

(a) Perpendicular (e-e) tensor elements

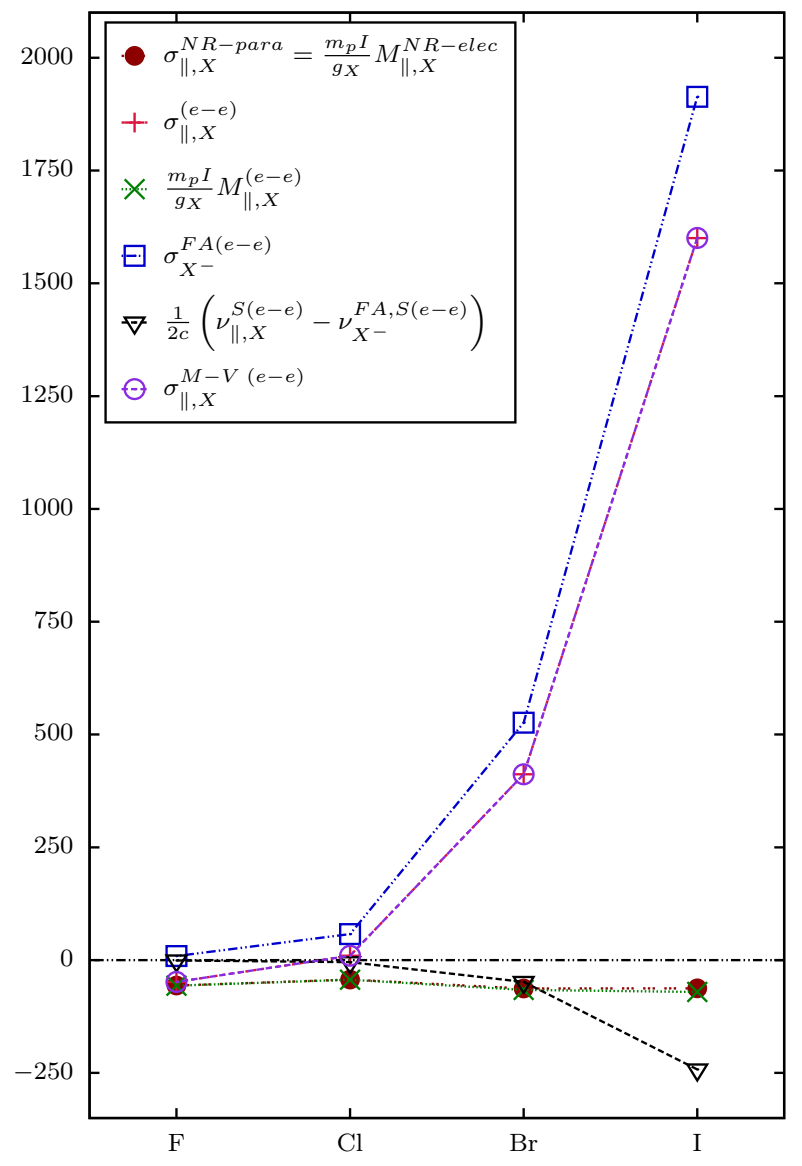

(b) Parallel (e-e) tensor elements

FIG. 1: Perpendicular and parallel contributions (in ppm) to $\boldsymbol{\sigma}_{X}^{(e-e)}, \boldsymbol{M}_{X}^{(e-e)}, \boldsymbol{\sigma}_{X}^{F A(e-e)}$ and $\boldsymbol{\nu}_{X}^{S(e-e)}-\boldsymbol{\nu}_{X}^{F A, S(e-e)}$ for the $X$ nuclei in $\mathrm{CH}_{3} X$ molecules $(X=\mathrm{F}, \mathrm{Cl}, \mathrm{Br}, \mathrm{I})$. Calculations were performed at the relativistic RPA level of approach. Values of $\boldsymbol{\sigma}_{X}^{N R-\text { para }}$ are also displayed.

with opposite signs (see Table III). Then the value of $\sigma_{i s o}^{(p-p)}-\sigma_{i s o}^{M-V(p-p)}$ is highly reduced, as happens when calculations are performed within the NR regime [17, 18].

An additional point must be raised here. The error introduced by including NMR shieldings of anions instead of the shielding of neutral free atoms, as it should be, can be estimated working within the NR regime. As an example, for iodine such a difference is found to be between -5.3 and $-5.1 \mathrm{ppm}$, according to the level of theory employed (see Supplemental Material [60]). Even though such differences (calculation of anions instead of neutral atoms) do not change the main statements pointed out above, they do contribute to a better reproduction of isotropic values by employing the model $\mathrm{M}-\mathrm{V}$.

\section{Isotropic shieldings and spans of halogens}

The application of model M-V give new and powerful tools to the analysis of the electronic origin of the relativistic effects on $\sigma_{i s o, X}$. As we did above we shall analyze now the (e-e) and (p-p) contributions to it.

First of all we should highlight here that $\sigma_{i s o, X}^{(e-e)}$ represents only up to $15 \%$ of the total shielding for iodine $(7 \%$ for bromine, $23 \%$ for chlorine and $10 \%$ for fluorine). This means that the (p-p) term gives the largest contribution, being its $M_{i s o, X}^{(p-p)}$ values almost zero in all cases, as expected [22, 66]. Besides, as stated recently in Ref. [15], the differences $\nu_{i s o, X}^{S(p-p)}-\nu_{X}^{F A, S(p-p)}$ are also vanishingly small. Therefore, $\sigma_{i s o, X}^{M-V(p-p)} \approx$ $\frac{m_{p}}{3 g_{X}} \operatorname{Tr}\left(\boldsymbol{M}_{X}^{n u c} \otimes \boldsymbol{I}\right)+\sigma_{X}^{F A(p-p)}$, being this expression close to the Ramsey-Flygare relationship for $\sigma_{i s o, X}^{N R, d i a}[17,18]$. Furthermore, almost all (more than 99\%) of the relativistic effects of $\sigma_{i s o, X}^{(p-p)}$ come from the relativistic effects of $\sigma_{X}^{F A(p-p)}$. These effects become larger as $\mathrm{Z}_{X}$ increase, being less than $13 \%$ in the case of iodine.

The NR limits of both, $\sigma_{i s o, X}^{(e-e)}$ and $M_{i s o, X}^{L(e-e)}$ fulfill the 
Ramsey-Flygare relationship but their corresponding relativistic effects have opposite signs. This is what underlies the breakdown of that well-known relation when heavy atoms are involved. On the other hand, the values of $\sigma_{i s o, X}^{M-V}(e-e)$ have a very good agreement with those of $\sigma_{i s o, X}^{(e-e)}$. This is so because the M-V model includes the contributions (from largest to smallest) of $\sigma_{X}^{F A(e-e)}$, $M_{i s o, X}^{S(e-e)}$ and $\nu_{i s o, X}^{S(e-e)}-\nu_{X}^{F A, S(e-e)}$, which are all zero in the NR limit. It is worth to stress that all of them are important in order to get the values of $\sigma_{i s o, X}^{M-V}(e-e)$ close to $\sigma_{i s o, X}^{(e-e)}$ (see Supplemental Material [60]). In the case of iodine, for instance, when $\sigma_{X}^{F A(e-e)}$ and $\nu_{i s o, X}^{S(e-e)}-\nu_{X}^{F A, S(e-e)}$ are neglected, $95 \%$ of the relativistic effects of $\sigma_{i s o, I}^{(e-e)}$ are not included. This means that the relativistic effects of $M_{i s o, I}^{(e-e)}$ represent only around $5 \%$ of the relativistic effects of $\sigma_{i s o, I}^{(e-e)}$. Therefore, the inadequate replacement of $M_{i s o, X}^{N R-p a r a}$ by $M_{i s o, X}^{(e-e)}$ as equivalent to the NR Ramsey-Flygare relationship (a proposal recently adopted in several works), gives incomplete and so, wrong results [4, 6, 13, 67].

We start now the analysis of correlation effects in $\sigma_{i s o, X}$ employing the M-V model. Among $97.0 \%$ and $101.5 \%$ of the correlation effects on both, $\sigma_{i s o, X}^{(e-e)}$ and $M_{i s o, X}^{L(e-e)}$ have a similar electronic origin (see Supplemental Material [60]).

This means that, when electron correlation effects are accurately included in $M_{X}^{(e-e)}$, much of these effects will be included in $\sigma_{i s o, X}^{(e-e)}$. On the other hand, correlation effects for $\sigma_{X}^{F A(p-p)}$, which are the main contributions to $\sigma_{i s o, X}^{(p-p)}$, are smaller than $0.5 \%$ (see Supplemental Material $[60])$.

All these findings can be summarized as follows: (i) relativistic effects in $M_{i s o, X}^{(e-e)}$ represent only a small fraction of its contributions to $\sigma_{i s o, X}^{(e-e)}$, whereas most of them are due to $\sigma_{X}^{F A(e-e)}$; (ii) correlation effects are almost of the same value for $\frac{m_{p}}{3 g_{X}} \operatorname{Tr}\left(\boldsymbol{M}_{X}^{(e-e)} \otimes \boldsymbol{I}\right)$ and $\sigma_{i s o, X}^{(e-e)}$; (iii) $\sigma_{i s o, X}^{(p-p)}$ are mostly independent of electronic correlation effects, and (iv) relativistic effects on $\sigma_{i s o, X}^{(p-p)}$ are smaller than $13 \%$. Then, one can estimate the accuracy of model $\mathrm{M}-\mathrm{V}$ to reproduce the total values of isotropic shieldings of halogen atoms in methyl halides by knowing that $\frac{\sigma_{i s o, X}^{M-V}-\sigma_{i s o, X}}{\sigma_{i s o, X}}$ is smaller than 0.013 for fluorine, 0.006 for chlorine, 0.002 for bromine and 0.001 for iodine.

The analysis of the anisotropy of the shielding can be performed from the span $(\Omega)$ of the halogens nuclei in methyl halides (i.e. $\Omega_{X}=\sigma_{\perp, X}-\sigma_{\|, X}$ ) employing the model M-V. In Fig. 2 the accuracy of this model in reproducing the $4 \mathrm{c}$ values of the shielding's span is observed. The small differences between them are almost completely given by their ( $\mathrm{p}-\mathrm{p})$ contributions, as can be seen in Table III.

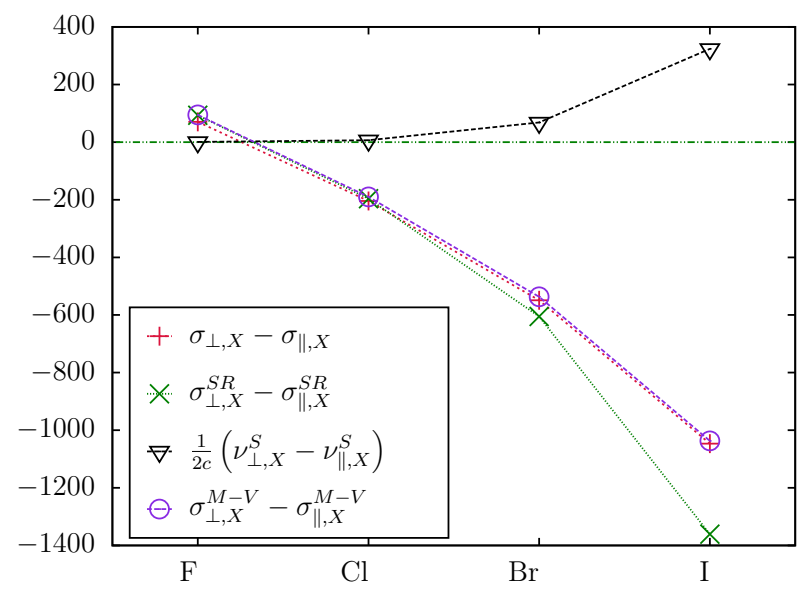

FIG. 2: 4c RPA values of the spans of NMR shielding,

SR tensors and results using model M-V (i.e., perpendicular minus parallel contributions to $\boldsymbol{\sigma}_{X}, \boldsymbol{M}_{X}$ and $\left.\boldsymbol{\sigma}_{X}^{M-V}\right)$ in $\mathrm{CH}_{3} X$ molecules $(X=\mathrm{F}, \mathrm{Cl}, \mathrm{Br}, \mathrm{I})$. All values are given in ppm.

Another fact that Fig. 2 highlights is the crucial role that $\nu_{X}^{S}$ plays in the reproduction of the $4 \mathrm{c}$ values of $\Omega_{X}$. The $4 \mathrm{c}-\mathrm{RPA}$ value of $\frac{1}{2 c}\left(\nu_{\perp, I}^{S}-\nu_{\|, I}^{S}\right)$ in methyl iodide is $323.87 \mathrm{ppm}$, whereas $\Omega_{I}=-1045.53 \mathrm{ppm}$ for this molecule. This not only indicates how important the relativistic effects are for accurately reproducing the values of spans (because $\nu^{S}$ is always zero in the NR limit), but even more important is that it confirms that $\nu^{S}$ cannot be neglected to accurately reproduce the complete NMR shielding tensor from its relation with the SR tensor.

\section{New semi-experimental absolute values of $\sigma_{X}$}

From the three terms of Eq. (10) only one, the first one, can be either taken from calculations or from experiments. Including its values from experiments, semiexperimental values of $\sigma_{i s o, X}$ are obtained and given in Table IV. The main differences among theoretical and experimental values of $\sigma_{i s o, X}^{S R}$ (see Eq. (6)), shown in the second column of Table IV, are mainly due to the discrepancies between experimental data and the calculated values of $M_{\perp, X}$ (and not $M_{\|, X}$, because $\left.I_{\perp}>I_{\|}\right)$. As observed in Table II the best agreement between $M_{\perp, X}^{\text {expt }}$ and their calculated counterparts is found for iodine in $\mathrm{CH}_{3} \mathrm{I}$ at the DFT/PBE0 level of theory, being the same behavior observed in Table IV.

On the other hand, the second term of Eq. (10) cannot be directly obtainable employing the DIRAC code because linear response calculations for open-shell electronic structures (as halogens are) are not currently implemented in it. Then, they can be estimated as the sum 
TABLE IV: Semi-experimental values of $\sigma_{i s o, X}$ obtained from experimental values of SR constants taken from Table II and 4c-DFT/PBE0 calculations of $\sigma_{X^{-}}^{F A}, \nu_{i s o, X}^{S}$ and $\nu_{X^{-}}^{F A, S}$ for halogens in methyl halides. Results in ppm.

\begin{tabular}{|c|c|c|c|c|c|}
\hline & $\sigma_{\text {iso }, X}^{S R}(\operatorname{expt})^{\mathrm{a}}$ & $\sigma_{X^{-}}^{F A}$ & $\Delta \sigma_{X}^{F A, N R_{\mathrm{b}}}$ & $\Delta \nu_{X}^{S \mathrm{c}}$ & $\sigma_{i s o, X}^{M-V}($ semi-expt $)$ \\
\hline $\mathrm{Br}$ & $\begin{array}{c}-596.28 \pm 3.98 \\
{[-606.43]^{\mathrm{d}}}\end{array}$ & 3439.70 & -6.08 & -2.73 & $\begin{array}{c}2834.61 \pm 3.98 \\
{[2825.92]^{\mathrm{e}}[2460.2]^{\mathrm{g}}}\end{array}$ \\
\hline I & $\begin{array}{c}-1280.21 \pm 2.42 \\
{[-1281.90]^{\mathrm{d}}}\end{array}$ & 6800.30 & -5.28 & -25.69 & $\begin{array}{c}5489.12 \pm 2.42 \\
{[5487.96]^{\mathrm{e}}[4261.3]^{\mathrm{g}}}\end{array}$ \\
\hline
\end{tabular}

${ }^{\text {a }}$ Obtained as $\frac{m_{p}}{3 g_{X}} \operatorname{Tr}\left(\boldsymbol{M}_{X}^{\text {expt }} \otimes \boldsymbol{I}\right)$, where $\boldsymbol{M}_{X}^{\text {expt }}$ are taken from Table II.

b $\Delta \sigma_{X}^{F A, N R}=\sigma_{X}^{F A, N R}-\sigma_{X-}^{F A, N R}$ is the difference between NR/PBE0 shielding of neutral and ionized atom (see Supplemental Material $[60])$.

${ }^{\mathrm{c}} \Delta \nu_{X}^{S}=\frac{1}{2 c}\left(\nu_{\text {iso }, X}^{S}-\nu_{X^{-}}^{F A, S}\right)$.

${ }^{\mathrm{d}}$ Four-component PBE0 calculation (see Supplemental Material [60]).

e Four-component PBE0 calculation of $\sigma_{\text {iso,X }}$ (see Supplemental Material [60]).

${ }^{\mathrm{f}}$ Experimental value taken from Ref. [37].

g Four-component BP86 calculation of $\sigma_{X}^{\text {dia }}+\sigma_{X}^{S R}$. Taken from Ref. [13].

of two terms: (i) the shielding of the ionized closed-shell atom instead of the neutral atom, i.e. $\sigma_{X^{-}}^{F A}$, and (ii) the NR correction given by $\Delta \sigma_{X}^{F A, N R}=\sigma_{X}^{F A, N R}-\sigma_{X^{-}}^{F A, N R}$, where $\sigma^{F A, N R}$ is calculated as a NR electronic groundstate expectation value.

Concerning how important are electron correlation effects on the absolute values of the shieldings one should first realize that the correlation effects are naturally included in experimental data. So, the semi-experimental values of absolute NMR shieldings given in Table IV do include most of such effects.

The way to include relativistic effects requires an special analysis. This will also shed some light on the reasons of the differences between our results with the ones recently published by Uhlíková and Urban [13]. In order to find the absolute isotropic shielding constants of bromine and iodine they calculated $\sigma_{i s o, X}^{(e-e)}, \sigma_{i s o, X}^{(p-p)}$ and $\frac{m_{p}}{3 g_{X}} \operatorname{Tr}\left(\boldsymbol{M}_{X}^{\text {elec }} \otimes \boldsymbol{I}\right)$ (in their work, $\sigma^{\text {para }}, \sigma^{\text {dia }}$ and $\sigma^{S R}$, respectively) at 4c-DFT/BP86 level of theory and employing $a b$ initio optimized geometries. They also obtained NR values by scaling the speed of light 100 times its real value. Even though their results were obtained using geometries and basis sets other than those employed in the present work, an appropriate comparison with ours are still valid. In their Table 5, they compare calculations of $\sigma_{i s o, X}$ (i.e. $\sigma_{i s o, X}^{(e-e)}+\sigma_{i s o, X}^{(p-p)}$ ) and $\frac{m_{p}}{3 g_{X}} \operatorname{Tr}\left(\boldsymbol{M}_{X}^{\text {elec }} \otimes \boldsymbol{I}\right)+\sigma_{i s o, X}^{(p-p)}$ with values of shieldings mentioned as "experimental", being them obtained by the application of the NR Ramsey-Flygare relationship (our Eq. (4)) and including experimental SR constants [30, 31]. They stated that Eq. (4) "can be used to determine the absolute nuclear shielding us- ing a specific correction value, which will depend on the position of an element in the periodic table" [13]. Such a correction value should be related to $\sigma_{i s o, X}^{(e-e)}-$ $\frac{m_{p}}{3 g_{X}} \operatorname{Tr}\left(\boldsymbol{M}_{X}^{\text {elec }} \otimes \boldsymbol{I}\right)$, and following Eq. (8) of our model this is equal to $\sigma_{X}^{F A(e-e)}+\frac{1}{2 c}\left(\nu_{i s o, X}^{S(e-e)}-\nu_{X}^{F A, S(e-e)}\right)$ (note that according to Ref. [22], $\boldsymbol{M}_{X}^{\text {elec }(p-p)}$ is almost negligible in all cases). Then, this "specific correcting value" should be related to an atomic contribution $\left(\sigma_{X}^{F A(e-e)}-\frac{1}{2 c} \nu_{X}^{F A, S(e-e)}\right)$ together with another term that depends on the environment $\left(\frac{1}{2 c} \nu_{i s o, X}^{S(e-e)}\right)[15,16,26]$.

The calculated values of $\frac{m_{p}}{3 g_{X}} \operatorname{Tr}\left(\boldsymbol{M}_{X}^{\text {elec }} \otimes \boldsymbol{I}\right)+\sigma_{i s o, X}^{(p-p)}$ published in Ref. [13] are closer to the experimental values of $\sigma_{i s o, X}^{e x p}$ published long time ago in Ref. 30 and Ref. 31 , than $\sigma_{i s o, X}^{(e-e)}+\sigma_{i s o, X}^{(p-p)}$. Nevertheless, this is in contrast with our findings because our calculated values of $\sigma_{\text {iso,X }}$ are in very good agreement with $\sigma_{i s o, X}^{M-V}$ (semi-expt), and far from $\frac{m_{p}}{3 g_{X}} \operatorname{Tr}\left(\boldsymbol{M}_{X}^{\text {elec }} \otimes \boldsymbol{I}\right)+\sigma_{\text {iso,X }}^{(p-p)}$ (see Table IV and Supplemental Material [60]).

According to this analysis the model M-V is useful not only for obtaining semi-experimental absolute values of NMR shieldings, but also to learn more about the physics that is behind such magnetic property.

\section{E. Absolute shieldings of ${ }^{13} \mathrm{C}$}

First of all we shall consider the dependence on the shielding of carbon atoms when the isotope ${ }^{13} \mathrm{C}$ replace that of ${ }^{12} \mathrm{C}$ in our calculations.

One difference may arise by the modification of the 
gauge origin position (see Eq. (3). In the present work, the gauge origin was placed at the molecular center of mass, which will change only a bit with the switch from ${ }^{12} \mathrm{C}$ to ${ }^{13} \mathrm{C}$. Then, the shielding values are almost the same independently of which carbon isotope is used. Furthermore, when the GIAO approach is used instead of the CGO one, the gauge origin dependence for the NMR shieldings disappears and so they do not depend on which carbon's isotope is employed. Besides, the substitution of ${ }^{12} \mathrm{C}$ by ${ }^{13} \mathrm{C}$ will slightly change the values reported above for tensor $\boldsymbol{M}$ of halogen and hydrogen atoms. Such differences are expected to happen because of the small displacement of the position of the molecular center of mass due to the isotope switch. The mentioned isotopic dependence clearly appears in both $\boldsymbol{M}^{n u c}$ and $\boldsymbol{M}^{\text {elec }}$ (see Eq. (1) and Eq. (2)).

On the other hand, relativistic effects for both, (e-e) and ( $\mathrm{p}-\mathrm{p})$ contributions to the shielding of carbons in methyl halides are almost independent of the amount of electron correlation involved. When relativistic effects are computed at RPA level of approach for $\sigma_{i s o, C}^{(e-e)}$, their results $(34.85 \mathrm{ppm})$ are close to those computed at KT3, BP86 and PBE0 levels (33.04 ppm, $30.81 \mathrm{ppm}$ and $29.97 \mathrm{ppm}$, respectively). In addition, relativistic effects for $\sigma_{i s o, C}^{(p-p)}$ at the RPA level of approach $(-1.51 \mathrm{ppm}$ for $\mathrm{CH}_{3} \mathrm{I}$ ) are almost the same as those obtained within the DFT approach (between $-1.54 \mathrm{ppm}$ and $-1.50 \mathrm{ppm}$ ).

Furthermore, as observed in Fig. 3 almost all relativistic effects on $\boldsymbol{\sigma}_{C}$ and $\boldsymbol{M}_{C}$ arise from $\sigma_{\perp, C}^{(e-e)}$ and $M_{\perp, C}^{(e-e)}$. Considering the RPA level of approach, relativistic effects in $\sigma_{\perp, C}^{(e-e)}$ for methyl iodide represent $-65.7 \%$ of its total value. That proportion goes down as the halogen atom becomes lighter: $-12.5 \%$ for $\mathrm{CH}_{3} \mathrm{Br},-3.2 \%$ for $\mathrm{CH}_{3} \mathrm{Cl}$ and $-1.2 \%$ for $\mathrm{CH}_{3} \mathrm{~F}$. From here it appears evident that those relativistic effects grows up as $Z_{X}$ increases.

There is another feature that one can realize by observing Fig. 3: $\sigma_{\perp, C}^{(e-e)}$ and $\frac{m_{p} I}{g_{C}} M_{\perp, C}^{(e-e)}$ are close each other. Then, they almost fulfill the NR Ramsey-Flygare relationship. This trend indicates that the addition of the following terms, $\sigma_{C}^{F A(e-e)}+\frac{1}{2 c}\left(\nu_{\perp, C}^{S(e-e)}-\nu_{C}^{F A, S(e-e)}\right)$ has a small contribution in methyl halides. So, the values of the difference $\sigma_{\perp, C}^{(e-e)}-\frac{m_{p} I}{g_{C}} M_{\perp, C}^{(e-e)}$ are always between $-1.6 \mathrm{ppm}$ and $2.5 \mathrm{ppm}$.

Given that linear response calculations for open-shell systems (as carbon atom is) are not implemented in the DIRAC code, one can estimate $\sigma_{C}^{F A}$ from its NR value. Then, the way to calculate $\sigma_{C}^{F A, N R}$ is through the NR limit of $\frac{1}{3 c_{0}^{2}}\left\langle 0\left|\frac{1}{\left|\boldsymbol{r}-\boldsymbol{R}_{C}\right|}\right| 0\right\rangle$, where $|0\rangle$ denotes the electronic DHF ground state of the atom. For getting such expectation value the NSTDIAMAGNETIC keyword of the DIRAC program must be used. In the present work the NR limit was reached by considering $c=100 c_{0}$, and doing it we found that $\sigma_{C}^{F A, N R}=260.53 \mathrm{ppm}$. Furthermore, the paramagnetic contributions to $\sigma^{F A, N R}$ are zero within the NR regime, being such contributions obtained as the

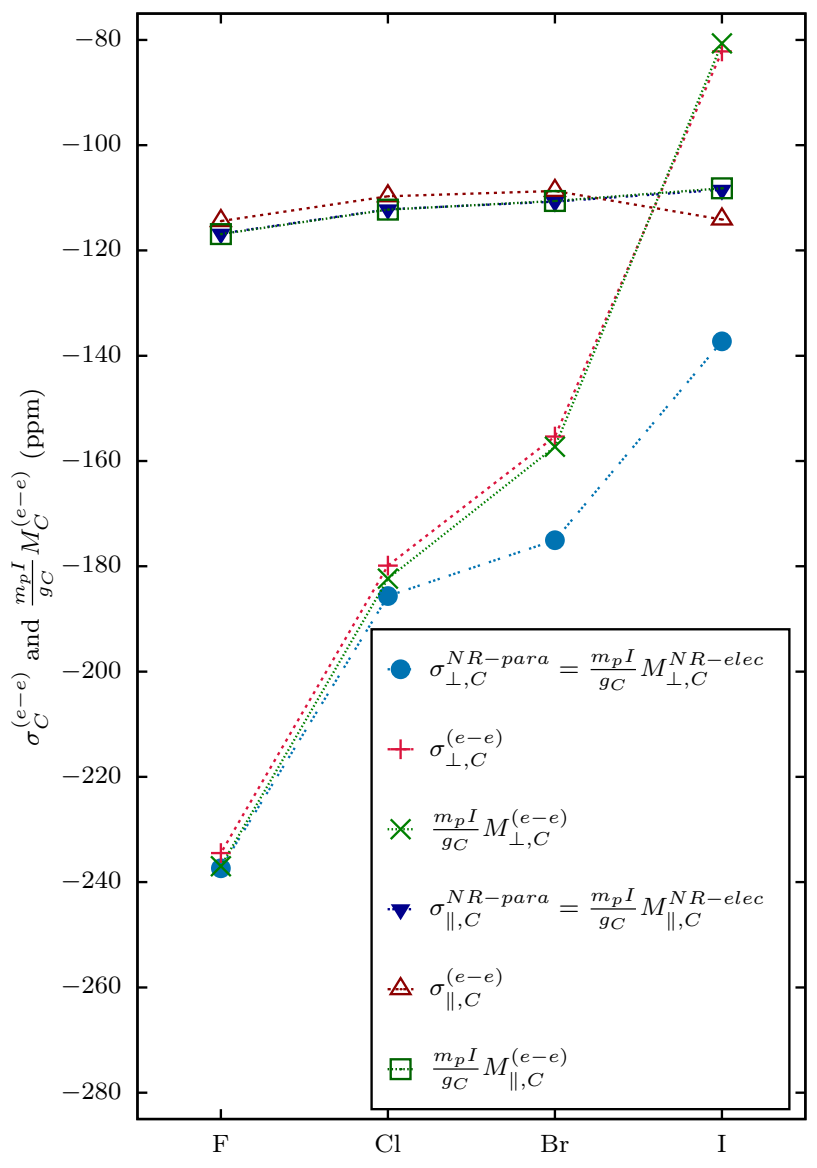

FIG. 3: Relativistic RPA values of the perpendicular and parallel contributions to $\boldsymbol{\sigma}_{C}^{(e-e)}$ and $\boldsymbol{M}_{C}^{(e-e)}$ in $\mathrm{CH}_{3} X$ molecules $(X=\mathrm{F}, \mathrm{Cl}, \mathrm{Br}, \mathrm{I})$, together with their NR limits. Values are given in ppm.

NR limit of $\sigma^{F A(e-e)}$.

Concerning the addition of electron correlation and relativistic effects on the (p-p) contribution to the carbon shieldings, it can be shown (see Eqs. (9) and (10)) that calculations of

$\Delta_{i s o, C}^{(p-p)}=\sigma_{i s o, C}^{(p-p)}-\frac{m_{p}}{3 g_{C}} \operatorname{Tr}\left[\left(\boldsymbol{M}_{C}^{n u c}+\boldsymbol{M}_{C}^{(p-p)}\right) \otimes \boldsymbol{I}\right]-\frac{1}{2 c} \nu_{i s o, C}^{S(p-p)}$

give results that belong to the range $(261.4 \pm 2.7) \mathrm{ppm}$ for all methyl halides and all methods (see Supplemental Material [60]). In addition to that it was shown in Ref. [15] that $\frac{1}{2 c} \nu^{F A, S(p-p)}$ is almost equal to $\frac{1}{2 c} \nu_{\text {iso }}^{S(p-p)}$. As $\frac{1}{2 c} \nu_{i s o, C}^{S(p-p)}=-0.35 \mathrm{ppm}$ for all methyl halides at both RPA and DFT levels of approach, this means that, according to the model M-V,$\sigma_{C}^{F A(p-p)}$ should belongs to the range $(261.0 \pm 2.7) \mathrm{ppm}$. Therefore, comparing the latter results with those of $\sigma_{C}^{F A, N R}(=260.53 \mathrm{ppm})$ we found that the combined electron correlation and relativistic effects in $\sigma_{C}^{F A(p-p)}$ are smaller than $3.2 \mathrm{ppm}$.

All this shows an additional advantage of using the model M-V. It gives another way to estimate relativistic 
effects for shieldings of nuclei in free atoms with high accuracy, i.e., from molecular calculations of $\boldsymbol{\sigma}$ and $\boldsymbol{M}$. Some time ago few attempts were made to calculate shieldings of free atoms [68-70], but given that 4c linear response calculations for open-shell electronic structures are not still implemented in relativistic codes, only shieldings of neutral noble gases were obtained, as well as shieldings of ionized atoms with closed-shell structures. The model M-V allows for accurate estimations of NMR shieldings for all kind of neutral atoms.

\section{CONCLUDING REMARKS}

Some of our previous theoretical works were focused on the development of formalisms aimed to best calculate absolute shielding scales by extending the well-known non relativistic Ramsey-Flygare rule to the relativistic regime. Its first and successful applications were made on linear molecules. In this work we did one step further applying that formalism to non-linear systems.

Systematic and highly accurate four-component, 4c, calculations of spin-rotation, SR, tensors and NMR magnetic shieldings were performed at RPA and DFT levels of theory for all nuclei of methyl halides. A comparison with experimental values of the SR tensor shows that theoretical expressions proposed in Ref. [22] accurately describe this spectroscopic parameter. It was found that, even though the electron correlation and relativistic effects in SR tensors are not of the same order of magnitude, both effects must be introduced simultaneously to accurately describe the behavior of the experimental data.

Some of the most important findings of this work are the following:

1. Experimental measurements of spin-rotation tensors of nuclei that belongs to methyl halides can be accurately reproduced by theoretical calculations.

2. The $\mathrm{M}-\mathrm{V}$ model reproduces 4c calculations of shieldings of $\mathrm{H}, \mathrm{C}, \mathrm{F}, \mathrm{Cl}, \mathrm{Br}$ and I with high accuracy (at both levels of approach, RPA and DFT). This includes the 4c calculation of SR constants, $\boldsymbol{\sigma}^{F A}$ and $\boldsymbol{\nu}^{S}-\boldsymbol{\nu}^{F A, S}$. Then, this model can be safely employed to obtain semi-experimental values of $\boldsymbol{\sigma}$ by the combination of experimental SR data with the calculation of $\boldsymbol{\sigma}^{F A}$ and $\boldsymbol{\nu}^{S}-\boldsymbol{\nu}^{F A, S}$. These last values are more accurate for the heaviest atoms.

3. Most of the electron correlation effects for the shieldings of halogen nuclei, taken as the difference between DFT and RPA calculations, are strongly related with those effects in SR constants. Therefore, when experimental data for SR are used, much of these effects in shieldings are accurately taken into account.

4. Relativistic effects of both properties, $\boldsymbol{M}$ and $\boldsymbol{\sigma}$, are negligible small for hydrogen nuclei in methyl halides.

5. A comparison between experimental values and $4 \mathrm{c}$ calculations of $M_{\|, C l}$ at different levels of approach shows that available experimental data for this parameter needs to be revisited.

6. We found a new procedure to estimate the free atom NMR shieldings. This procedure requires the calculation of $\boldsymbol{M}$ and $\boldsymbol{\sigma}$ of the given atom in a set of different molecules.

Another important finding is the fact that the results of previous models (see for instance Ref. [4], [6], [67] and [13]), which consider the sSR constants and the calculation of $(p-p)$ contributions to the shieldings (its diamagnetic-like contributions) should be taken with caution. They usually do not take into account the contributions of $\boldsymbol{\sigma}^{F A(e-e)}$ and $\boldsymbol{\nu}^{S}-\boldsymbol{\nu}^{F A, S}$ which may be so large that must be included. In addition, it should be pointed out that their proposal of an atomic correction may not be correct, because $\boldsymbol{\nu}^{S}$ depends on the symmetry of the molecule and it can be not negligible at all.

\section{ACKNOWLEDGMENTS}

Support from CONICET by grant PIP 11220130100361 and FONCYT by grant PICT 2016-2936 are greatly acknowledged.

We thank the Institute for Modeling and Innovation on Technologies (IMIT) of the National Scientific and Technical Research Council and the Northeastern University of Argentina for their support and for providing access to the institutional high performance computing cluster.
[1] N. F. Ramsey, Phys. Rev. 78, 699 (1950).

[2] W. H. Flygare, J. Chem. Phys. 41, 793 (1964).

[3] S. S. Gomez and G. A. Aucar, J. Chem. Phys. 134, 204314 (2011).

[4] E. Malkin, S. Komorovský, M. Repisky, T. B. Demissie, and K. Ruud, J. Phys. Chem. Lett. 4, 459 (2013).

[5] Y. Xiao, Y. Zhang, and W. Liu, J. Chem. Theory Comput. 10, 600 (2014).
[6] K. Ruud, T. B. Demissie, and M. Jaszuński, J. Chem. Phys. 140, 194308 (2014).

[7] S. Komorovský, M. Repisky, E. Malkin, K. Ruud, and J. Gauss, J. Chem. Phys. 142, 091102 (2015).

[8] T. B. Demissie, M. Jaszuński, E. Malkin, S. Komorovský, and K. Ruud, Mol. Phys. 113, 1576 (2015).

[9] S. Komorovský, M. Repisky, E. Malkin, T. B. Demissie, and K. Ruud, J. Chem. Theory Comput. 11, 3729 (2015), 
pMID: 26574455.

[10] T. B. Demissie, M. Jaszuński, S. Komorovský, M. Repisky, and K. Ruud, J. Chem. Phys. 143, 164311 (2015).

[11] T. B. Demissie, Phys. Chem. Chem. Phys. 18, 3112 (2016).

[12] Y. Xiao, W. Liu, and K. Ruud, Relativistic Theory of Nuclear Spin-Rotation Tensor, in Handbook of Relativistic Quantum Chemistry, edited by W. Liu (Springer Berlin Heidelberg, Berlin, Heidelberg, 2017) pp. 693-723.

[13] T. Uhlíková and Š. Urban, Mol. Phys. 116, 1192 (2017).

[14] I. A. Aucar, S. S. Gomez, C. G. Giribet, and G. A. Aucar, Phys. Chem. Chem. Phys. 18, 23572 (2016).

[15] I. A. Aucar, S. S. Gomez, C. G. Giribet, and G. A. Aucar, J. Phys. Chem. Lett. 7, 5188 (2016).

[16] G. A. Aucar and I. A. Aucar, in Annual Rep. on NMR Spect., Vol. 96, edited by G. A. Webb (Academic Press, 2019) Chap. 3, pp. 77-141.

[17] W. H. Flygare and J. Goodisman, J. Chem. Phys. 49, 3122 (1968).

[18] T. D. Gierke and W. H. Flygare, J. Am. Chem. Soc. 94, 7277 (1972).

[19] W. H. Flygare, Chem. Rev. 74, 653 (1974).

[20] L. Visscher, T. Enevoldsen, T. Saue, H. J. A. Jensen, and J. Oddershede, J. Comput. Chem. 20, 1262 (1999).

[21] M. A. M. Forgeron, R. E. Wasylishen, and G. H. Penner, J. Phys. Chem. A 108, 4751 (2004).

[22] I. A. Aucar, S. S. Gomez, M. C. Ruiz de Azúa, and C. G. Giribet, J. Chem. Phys. 136, 204119 (2012).

[23] J. I. Melo, M. C. Ruiz de Azúa, C. G. Giribet, G. A. Aucar, and R. H. Romero, J. Chem. Phys. 118, 471 (2003).

[24] G. A. Aucar, J. I. Melo, I. A. Aucar, and A. F. Maldonado, Int. J. Quantum Chem. 118, e25487 (2018).

[25] I. A. Aucar, Ph.D. thesis, Universidad Nacional del Nordeste (2015).

[26] I. A. Aucar, C. A. Giménez, and G. A. Aucar, RSC Adv. 8, 20234 (2018).

[27] S. C. Wofsy, J. S. Muenter, and W. Klemperer, J. Chem. Phys. 55, 2014 (1971).

[28] S. G. Kukolich and A. C. Nelson, J. Am. Chem. Soc. 95, 680 (1973).

[29] A. Dubrulle, D. Boucher, J. Burie, and J. Demaison, Chem. Phys. Lett. 45, 559 (1977).

[30] J. Demaison, A. Dubrulle, D. Boucher, and J. Burie, J. Chem. Phys. 67, 254 (1977).

[31] D. Boucher, J. Burie, D. Dangoisse, J. Demaison, and A. Dubrulle, Chem. Phys. 29, 323 (1978).

[32] A. Dubrulle, J. Burie, D. Boucher, F. Herlemont, and J. Demaison, J. Mol. Spec. 88, 394 (1981).

[33] G. Wlodarczak, D. Boucher, R. Bocquet, and J. Demaison, J. Mol. Spec. 124, 53 (1987).

[34] S. Carocci, A. Di Lieto, A. De Fanis, P. Minguzzi, S. Alanko, and J. Pietila, J. Mol. Spec. 191, 368 (1998).

[35] L. N. Stříteská, M. Šimečková, P. Kania, P. Musil, L. Kolesniková, J. Koubek, and Š. Urban, J. Mol. Struct. 919, 89 (2009).

[36] G. Cazzoli and C. Puzzarini, J. Phys. Chem. A 119, 1765 (2015).

[37] K. Jackowski, M. Kubiszewski, and W. Makulski, J. Mol. Struct. 614, 267 (2002).

[38] K. Jackowski, M. Kubiszewski, and M. Wilczek, Chem. Phys. Lett. 440, 176 (2007).

[39] M. Wilczek, M. Kubiszewski, and K. Jackowski, J. Mol. Struct. 704, 311 (2004).
[40] P. Garbacz, K. Jackowski, W. Makulski, and R. E. Wasylishen, J. Phys. Chem. A 116, 11896 (2012).

[41] A. A. Cheremisin and P. V. Schastnev, J. Mag. Res. (1969) 40, 459 (1980).

[42] H. Nakatsuji, H. Takashima, and M. Hada, Chem. Phys. Lett. 233, 95 (1995).

[43] V. G. Malkin, O. L. Malkina, and D. R. Salahub, Chem. Phys. Lett. 261, 335 (1996).

[44] S. K. Wolff and T. Ziegler, J. Chem. Phys. 109, 895 (1998).

[45] J. Vaara, K. Ruud, O. Vahtras, H. Ågren, and J. Jokisaari, J. Chem. Phys. 109, 1212 (1998).

[46] J. Vaara, K. Ruud, and O. Vahtras, J. Chem. Phys. 111, 2900 (1999).

[47] J. I. Melo, M. C. Ruiz de Azúa, C. G. Giribet, G. A. Aucar, and P. F. Provasi, J. Chem. Phys. 121, 6798 (2004).

[48] A. M. Kantola, P. Lantto, J. Vaara, and J. Jokisaari, Phys. Chem. Chem. Phys. 12, 2679 (2010).

[49] D. O. Samultsev, Y. Y. Rusakov, and L. B. Krivdin, Russ. Chem. Bull. 64, 2756 (2015).

[50] D. O. Samultsev, Y. Y. Rusakov, and L. B. Krivdin, Magn. Reson. Chem. 54, 787 (2016).

[51] G. A. Aucar and J. Oddershede, Int. J. Quantum Chem. 47, 425 (1993).

[52] G. A. Aucar, T. Saue, L. Visscher, and H. J. A. Jensen, J. Chem. Phys. 110, 6208 (1999).

[53] W. Haynes, CRC Handbook of Chemistry and Physics, 97th Edition (CRC Press, 2016).

[54] P. Raghavan, At. Data Nucl. Data Tables 42, 189 (1989).

[55] DIRAC, a relativistic ab initio electronic structure program, Release DIRAC19 (2019), written by A. S. P. Gomes, T. Saue, L. Visscher, H. J. Aa. Jensen, and R. Bast, with contributions from I. A. Aucar, V. Bakken, K. G. Dyall, S. Dubillard, U. Ekström, E. Eliav, T. Enevoldsen, E. Faßhauer, T. Fleig, O. Fossgaard, L. Halbert, E. D. Hedegård, B. Heimlich-Paris, T. Helgaker, J. Henriksson, M. Iliaš, Ch. R. Jacob, S. Knecht, S. Komorovský, O. Kullie, J. K. Lærdahl, C. V. Larsen, Y. S. Lee, H. S. Nataraj, M. K. Nayak, P. Norman, G. Olejniczak, J. Olsen, J. M. H. Olsen, Y. C. Park, J. K. Pedersen, M. Pernpointner, R. di Remigio, K. Ruud, P. Sałek, B. Schimmelpfennig, B. Senjean, A. Shee, J. Sikkema, A. J. Thorvaldsen, J. Thyssen, J. van Stralen, M. L. Vidal, S. Villaume, O. Visser, T. Winther, and S. Yamamoto (available at http://dx. doi.org/10.5281/zenodo.3572669, see also http://www. diracprogram.org).

[56] T. Saue, R. Bast, A. S. P. Gomes, H. J. A. Jensen, L. Visscher, I. A. Aucar, R. Di Remigio, K. G. Dyall, E. Eliav, E. Faßhauer, T. Fleig, L. Halbert, E. D. Hedegård, B. Helmich-Paris, M. Iliaš, C. R. Jacob, S. Knecht, J. K. Lærdahl, M. Lopez Vidal, M. K. Nayak, G. Olejniczak, J. M. H. Olsen, M. Pernpointner, B. Senjean, A. Shee, A. Sunaga, and J. N. P. van Stralen, J. Chem. Phys. 152, 204104 (2020).

[57] L. Visscher, Theor. Chem. Acc. 98, 68 (1997).

[58] K. G. Dyall, Theor. Chem. Acc. 135, 128 (2016).

[59] K. G. Dyall, Theor. Chem. Acc. 115, 441 (2006).

[60] See Supplemental Material at http://link.aps.org/ supplemental/10.1103/PhysRevA.number.number for detailed tables with calculations of $\boldsymbol{M}, \boldsymbol{\sigma}^{M-V}$ and $\boldsymbol{\sigma}$ of halides and carbon nuclei in methyl halides.

[61] L. Visscher and K. G. Dyall, At. Data Nucl. Data Tables 67, 207 (1997). 
[62] T. W. Keal and D. J. Tozer, J. Chem. Phys. 121, 5654 (2004).

[63] A. D. Becke, Phys. Rev. A 38, 3098 (1988).

[64] J. P. Perdew and Y. Wang, Phys. Rev. B 33, 8800 (1986), erratum: 34, 7406 (1986).

[65] C. Adamo and V. Barone, J. Chem. Phys. 110, 6158 (1999).

[66] I. A. Aucar, S. S. Gomez, J. I. Melo, C. G. Giribet, and
M. C. Ruiz de Azúa, J. Chem. Phys. 138, 134107 (2013). [67] S. Komorovský, M. Repisky, E. Malkin, T. B. Demissie, and K. Ruud, J. Chem. Theory Comput. 11, 3729 (2015).

[68] J. Vaara and P. Pyykkö, J. Chem. Phys. 118, 2973 (2003).

[69] P. Manninen, P. Lantto, J. Vaara, and K. Ruud, J. Chem. Phys. 119, 2623 (2003).

[70] S. S. Gomez, A. F. Maldonado, and G. A. Aucar, J. Chem. Phys. 123, 214108 (2005). 